\title{
Correspondências entre classificações fisiográficas e padrões espaciais de assembleias de aves e mamíferos no Pantanal Brasileiro
}

\author{
Rogério Rodrigues Faria ${ }^{1, \varpi ;}$; Rosana Talita Braga ${ }^{1}$; CAmila Leonardo Mioto²; Antonio \\ Conceição Paranhos Filho ${ }^{2}$; Franco Leandro Souza ${ }^{3} \&$ Luis Mauricio Bini ${ }^{1}$
}

1. Laboratório de Ecologia Teórica e Síntese, Departamento de Ecologia, ICB, Universidade Federal de Goiás. 2. Laboratório de Geoprocessamento para Aplicações Ambientais. Centro de Ciências Exatas e Tecnologia, Universidade Federal de Mato Grosso Do Sul. 3. Centro de Ciências Biológicas e da Saúde. Universidade Federal de Mato Grosso Do Sul.

\begin{abstract}
Resumo. Estudos prévios no Pantanal brasileiro sugerem que heterogeneidade fisiográfica poderia ser usada para delimitar diferentes sub-regiões. Todavia, a magnitude na qual essas regionalizações refletem padrões espaciais de variação na diversidade beta para diferentes grupos taxonômicos não foi testada até o momento. O objetivo deste trabalho foi avaliar se os esquemas de regionalização propostos para o Pantanal brasileiro descrevem adequadamente a diversidade beta para aves e mamíferos. A presença e ausência de cada espécie de mamíferos e aves foram registradas em cada uma das 80 células de $0.5 \times 0.5$ graus em um mapa de grade quadriculada. O índice de similaridade de Jaccard foi usado para estimar a similaridade em cada par de células. Um método de classificação não hierárquico (k-means) foi aplicado para obter classificação "a posteriori" das células dentro de sub-regiões. As forças de classificações (CS) de cada classificação fisiográfica e "a posteriori" foi a diferença entre similaridade média dentro (W) e entre (B) subdivisões (CS=W-B). Ambas as classificações ("a priori" e "a posteriori") apresentaram baixos valores de CS, o que indica que elas não são aptas a representar os padrões de diversidade beta de mamíferos e aves dentro do Pantanal brasileiro. Os resultados sugerem que, provavelmente por causa da ampla distribuição geográfica e os baixos níveis de endemismo destes táxons, classificações fisiográficas do Pantanal brasileiro não são atalhos confiáveis para representar os padrões de diversidade beta de aves e mamíferos. Deste modo, inventários biológicos detalhados são necessários para a proposição de planos de conservação locais consistentes para esta região altamente diversa e ameaçada.
\end{abstract}

[Palavras-chave: regionalização; áreas úmidas; biodiversidade animal; k-means; táxons como substituto; forças de classificação]

\begin{abstract}
Aвstract. Correspondence between physiographic classifications and spatial patterns of $\beta$ diversity in mammal and bird species assemblages in the Brazilian Pantanal: Previous studies in the Brazilian Pantanal suggest that physiographic heterogeneity could be used to delimit different subregions. However, the extent to which these regionalisations reflect spatial patterns of variation in beta diversity for different taxonomic groups has not been tested yet. The aim of this paper was to evaluate whether current regionalization schemes proposed for the Brazilian Pantanal adequately describe patterns of beta diversity in mammals and birds. The presence-absence of each mammal and bird species was recorded at each of the eighty $0.5 \times 0.5$ degree cells in a squared grid map. A Jaccard similitude index was used to estimate the similarity between each pair of cells. A non-hierarchical classification method (k-means) was applied to obtain "a posteriori" classification of cells into subregions. The classification strength (CS) of each physiographic scheme and the "a posteriori" classification was the difference between mean similarity within (W) and between (B) subidivisions (CS=W-B). Both classifications ("a priori" and "a posteriori") showed low values of CS, which indicates that they were not suitable to represent the beta diversity patterns in mammals and birds within the Brazilian Pantanal. The results suggest that, probably because the large geographic ranges and low levels of endemism in these taxa, physiographic classifications of the Brazilian Pantanal are no reliable shortcuts to represent beta diversity patterns in mammal and birds. Thus, detailed biological inventories are necessary to propose consistent local conservation plans in this highly diverse and threatened region.
\end{abstract}

[Keywords: regionalization; wetlands; animal biodiversity; k-means; taxa as surrogate; classification strengths]

\section{INTRODUÇão}

A classificação geográfica (ou regionalização) consiste na partição de uma área em regiões menores relativamente homogêneas,

Coordenadoria de Relacionamento EmpresaUniversidade. Pró-Reitoria de Pesquisa e Pós-Graduação. Universidade Federal de Mato Grosso do Sul. Campo Grande; MS; Brasil. roger.faria@yahoo.com.br permitindo avaliar as variações espaciais de características biológicas e ambientais em diferentes escalas espaciais (Huang \& Ferng 1990). Essas regionalizações, com considerável implicação em estudos ecológicos, também

Editora Asociada: Adriana Ruggiero

Recibido: 29 de mayo de 2012; Fin de arbitraje: 27 de agosto; Segunda versión: 22 de octubre; Tercera versión: 22 de noviembre; Aceptado: 5 de diciembre. 
podem subsidiar importantes estratégias para conservação, manejo e monitoramento da biodiversidade (Rabeni \& Doisy 2000; Heino et al. 2002). Portanto, é esperado e requerido que um esquema de regionalização deva representar eficientemente a variabilidade da biodiversidade (Sandin \& Johnson 2000; Heino \& Mykrä 2006).

Em geral, esquemas de regionalização são derivados da delimitação de regiões com base em características fisiográficas obtidas em grandes escalas (Januchowski-Hartley et al. 2011). Uma elevada congruência entre um determinado esquema de classificação baseado em características fisiográficas e os padrões de diversidade beta (mudanças nas composições de espécies) permitiria usar esse esquema como substituto ("surrogate") da variação biológica (Leathwick et al. 2003; Bonn \& Gaston 2005). Do ponto de vista operacional e orçamentário, estas classificações baseadas em dados ambientais são as substitutas mais acessíveis (Arponen et al. 2008) e as mais comumente utilizadas (Heino \& Mykrä 2006; Andrew et al. 2011).

O Pantanal é a maior planície alagável do mundo, compreendendo uma área de aproximadamente $140000 \mathrm{~km}^{2}$, formado por depósitos sedimentares relativamente recentes (Ponce \& Cunha 1993). A planície pantaneira tem como limite leste o Cerrado, na porção nordeste, as florestas semidecíduas relacionadas com a floresta Amazônica e no sudoeste o Chaco (Adámoli 1982). Devido à sua história geomorfológica, à sua localização de fronteira com três grandes domínios fitogeográficos e também à influência dos seus principais rios (Paraguai, Bento Gomes, Cuiabá, São-Lourenço-Itiquira, Taquari, Negro, Aquidauana-Miranda, Nabileque e Apa), o Pantanal apresenta uma elevada diversidade de fitofisionomias e habitats, resultando em distintas sub-regiões (Silva 1995; Cunha \& Junk 1999; Silva et al. 2000). Devido às peculiaridades de cada região, é esperado que as mesmas demandem tratamento diferenciado para atividades produtivas, de manejo, conservação, delimitação e seleção de áreas de pesquisa, bem como para ações normativas ou legislativas (Silva \& Abdon 1998). Para algumas espécies animais, a ocorrência dentro de sub-regiões do Pantanal varia de acordo com a composição e distribuição local de espécies dos biomas vizinhos (Alho 2011).

A maior parte das espécies de animais que ocorrem no Pantanal tem ampla distribuição geográfica e, portanto, esse ambiente tem sido caracterizado por apresentar baixa freqüência de endemismos (e.g., Brown-Junior 1986; Lourival et al. 2000; Junk \& Cunha 2005; Junk et al. 2006). Contudo, a distribuição das espécies de animais dentro da planície pantaneira ainda é pouco conhecida (e.g., Carmignotto 2004; Camargo \& Fischer 2005; Longo et al. 2007). Apesar do crescente número de inventários faunísticos no Pantanal (ver Alho 2011), somente o estudo de Brown-Júnior (1986) tratou de questões zoogeográficas. Além disso, de acordo com as revisões de Silva (1995) e Locks et al. (2011), as classificações propostas para o Pantanal consideraram, até então, somente o aspecto abiótico e as formações vegetais. No entanto, a validação dessas classificações através do confronto com dados de distribuição da fauna não foi testada até o momento.

O objetivo principal deste trabalho foi avaliar se os esquemas de regionalização propostos para o Pantanal brasileiro descrevem adequadamente a diversidade beta para aves e mamíferos, grupos relativamente bem estudados na região. Embora haja controversa sobre a classificação geográfica para o Pantanal, há um consenso sobre a heterogeneidade ambiental dentro da planície de inundação a ponto de formar distintas subregiões (Silva 1995; Locks et al. 2011). No caso em que as classificações fisiográficas refletem adequadamente a variação na composição taxonômica de aves e mamíferos, cada sub-região englobará uma parte da variabilidade global da biodiversidade (Heino \& Mykrä 2006).

Estudos prévios têm demonstrado que os padrões de atividade e densidade de determinados grupos de vertebrados no Pantanal estão relacionados aos regimes de inundação e estrutura da paisagem (Mourão et al. 2000; Campos et al. 2003). Dada a influência do pulso de inundação sobre a dinâmica populacional da fauna do Pantanal (Alho 2008) e do papel dominante da vegetação na ocupação de habitats por espécies de vertebrados (Edwards et al. 1996), é esperado que os dados de aves e mamíferos estejam mais relacionados com as classificações que atendem aos critérios relacionados à vegetação e à hidrologia.

\section{Material e Métodos}

\section{Área de estudo}

O Pantanal brasileiro situa-se entre os paralelos $16^{\circ}$ e $21^{\circ} \mathrm{S}$ e os meridianos $55^{\circ}$ e $58^{\circ} \mathrm{W}$. Trata- 
se de uma planície sedimentar formada no quaternário, preenchida com depósitos aluviais dos rios da Bacia do Alto Paraguai (Mercante et al. 2011). A precipitação média anual é de $1396 \mathrm{~mm}$, apresentando um claro regime sazonal no ciclo hidrológico, identificando um período de chuva, entre outubro e abril, e um período de estiagem, nos demais meses (Gonçalves et al. 2011). Para a planície são reconhecidas 17 classes de vegetação, desde áreas de formações pioneiras até áreas de Savana Florestada, das quais a mais representativa é a Savana Arborizada com aproximadamente 36\% de abrangência (Silva et al. 2000). Em relação à hidrologia, os maiores rios que drenam o Pantanal são: Paraguai, Bento Gomes, Cuiabá, São-LourençoItiquira, Taquari, Negro, Aquidauana-Miranda, Nabileque e Apa (Alho 2008). Os rios de maior porte periodicamente transbordam ao longo da planície e, após certo período de inundação, uma mesma sub-bacia sofre influência da inundação das subbacias vizinhas (Silva 1995). As altitudes variam de 100 a 150 m (Mercante et al. 2011), com declividade de aproximadamente de 50 a $30 \mathrm{~cm} / \mathrm{km}$ no sentido leste-oeste e de 3 a $1.5 \mathrm{~cm} / \mathrm{km}$ de norte para o sul (Gonçalves et al. 2011). Baseando-se somente nos tipos de solos, Amaral-Filho (1986) reconheceu 13 tipos de "pantanais". A variação anual no nível da água, denominada por Junk \& Silva (1999) de pulso de inundação, caracteriza-se por períodos em que a cheia apresenta uma ascensão e uma recessão por ano, ocorre em ciclos plurianuais, com períodos de anos muito chuvosos e outros relativamente secos. Por fim, a planície pantaneira, pode ser inundada não só pelos rios que transbordam, mas também por elevação do nível d'água subterrânea e pelas chuvas (Gonçalves et al. 2011).

\section{As classificações para o Pantanal}

As principais classificações que serão testadas nesse estudo apresentam valores contrastantes tanto na área total delimitada $\left(50000 \mathrm{~km}^{2}\right.$ a $\left.130000 \mathrm{~km}^{2}\right)$ a, quanto no número de sub-regiões identificadas (11 a 18) (Tabela 1, Figura 1). De maneira geral, as propostas baseiam-se em critérios fisiográficos, tais como, características pedológicas, geológicas, geomorfológicas, hidrológicas e ecológicas (Silva 1995). De acordo com Silva \& Abdon (1998), os estudos fisiográficos do Pantanal são divididos entre aqueles associados ao Estudo de Desenvolvimento Integrado da Bacia do Alto Paraguai (EDIBAP) (Sanchez 1977; Brasil 1979; Adámoli 1982) e aqueles associados ao Projeto RADAM BRASIL (Franco \& Pinheiro 1982; Alvarenga et al. 1982, 1984; Amaral Filho 1986). Na visão de Silva \& Abdon (1998), as delimitações adotadas pelo grupo do RADAM BRASIL são as mais consistentes, por usarem diferentes características físicas e bióticas, como feições de relevo, drenagem, solos e vegetação. Pode ser observado que, mesmo dentro destas duas vertentes, não há um consenso quanto ao número de sub-regiões e ao padrão de sub-divisão do Pantanal. Para esse trabalho apenas seis das nove propostas foram utilizadas para as análises (Tabela 1, Figura 1). A proposta de Mato Grosso do Sul (1989) só atende ao Estado do Mato Grosso do Sul e assim a parte do Pantanal referente ao Estado de Mato Grosso não seria analisada. A classificação de Amaral-Filho (1986) não foi empregada, pois como é baseada em solos, as sub-regiões não tinham limites claros e em algumas situações há pequenos manchas de uma região disjuntos e/ou imerso em outra região. Por fim Sanchez (1977) não foi utilizado por não haver a delimitação do

Tabela 1. Propostas de delimitação do Pantanal Brasileiro, incluindo os critérios, fontes, número de sub-regiões e a área total delimitada.

Table 1. Subdivisions of the Brazilian Pantanal, including criteria, source, number of sub regions and total area.

\begin{tabular}{|c|c|c|c|c|}
\hline Autor & Critérios adotados & Material utilizado & $\begin{array}{l}\text { Número de } \\
\text { sub-regiões }\end{array}$ & $\begin{array}{l}\text { Área } \\
\left(\mathrm{km}^{2}\right)\end{array}$ \\
\hline Brasil (1979) & $\begin{array}{l}\text { Geomorfológico, hidrológico e } \\
\text { fluviomorfológico }\end{array}$ & $\begin{array}{l}\text { Imagens de Radar na escala 1:250000 } \\
\text { e cartas topográficas } \\
\text { Na escala 1:100000 }\end{array}$ & 15 & 139111 \\
\hline $\begin{array}{l}\text { Franco \& Pinheiro } \\
\text { (1982), Alvarenga } \\
\text { et al. (1982) }\end{array}$ & $\begin{array}{l}\text { Geomorfológico, fatores } \\
\text { morfogenéticos e cartas } \\
\text { topográficas (altimetria } \\
\text { relativa, litologia e pedologia) }\end{array}$ & $\begin{array}{l}\text { Imagens de radar na } \\
\text { escala 1:250000 }\end{array}$ & 13 & 136738 \\
\hline Adámoli (1982) & Fitogeográfico e hidrológico & $\begin{array}{l}\text { Estudos anteriores } \\
\text { EDIBAP. Imagens do } \\
\text { LANDSAT-MSS nas } \\
\text { escalas 1:250000 e 1:1000000 }\end{array}$ & 10 & 139111 \\
\hline $\begin{array}{l}\text { Alvarenga et al. } \\
\text { (1984) }\end{array}$ & $\begin{array}{l}\text { Geomorfológico e aspectos } \\
\text { estruturais topográficos, } \\
\text { hidrológicos, morfológicos, } \\
\text { pedológicos e de estrutura vegetal }\end{array}$ & $\begin{array}{l}\text { Imagens de Radar na } \\
\text { escala 1:250000. } \\
\text { Imagens de LANDSAT-MSS nas } \\
\text { escalas 1:500000 e 1:1000000 }\end{array}$ & 12 & 133465 \\
\hline Silva \& Abdon (1998) & $\begin{array}{l}\text { Aspectos fisiomorfológicos e } \\
\text { geopolíticos }\end{array}$ & $\begin{array}{l}\text { Estudos Anteriores, GPS e imagens } \\
\text { Landsat TM na } \\
\text { composição colorida, } \\
\text { escala 1:250000 }\end{array}$ & 11 & 138183 \\
\hline Locks et al. (2011) & Tipo de vegetação e inundação & $\begin{array}{l}\text { Imagens obtidas pelo sensor WFI, } \\
\text { satélite CBERS-2B }\end{array}$ & 18 & 136928 \\
\hline
\end{tabular}

Fonte: Adaptado de Silva (1995) e Locks et al. (2011) 


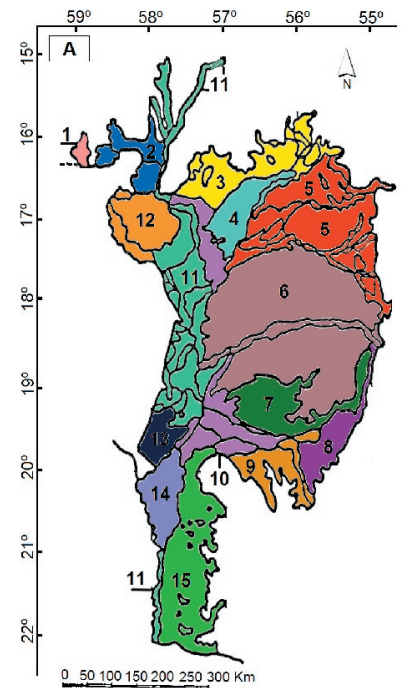

1 Pa. do Corixo Grande
2 Pa. do Jaurú-Cabaçal-Sepotuba 3 Gomedo Paraguaizinho-Bento 4 Pa. do Cuiabá-Cassange 5 Pa. de S. Lourenço-ltiquira6 Pa. do Taquari Fa. da Nhecolândia Pa. do Negro 9 Pa. de Miranda-Aquidauana 10 Plan. das vertentes do 11 Plan. de inundação do 12 Pa. do Descalvado-Uberabe i. Pa. de Jacadigo-Coimbra $14 \mathrm{~Pa}$. do Nabileque 15 Plan. Chaquenhas Deprimidas
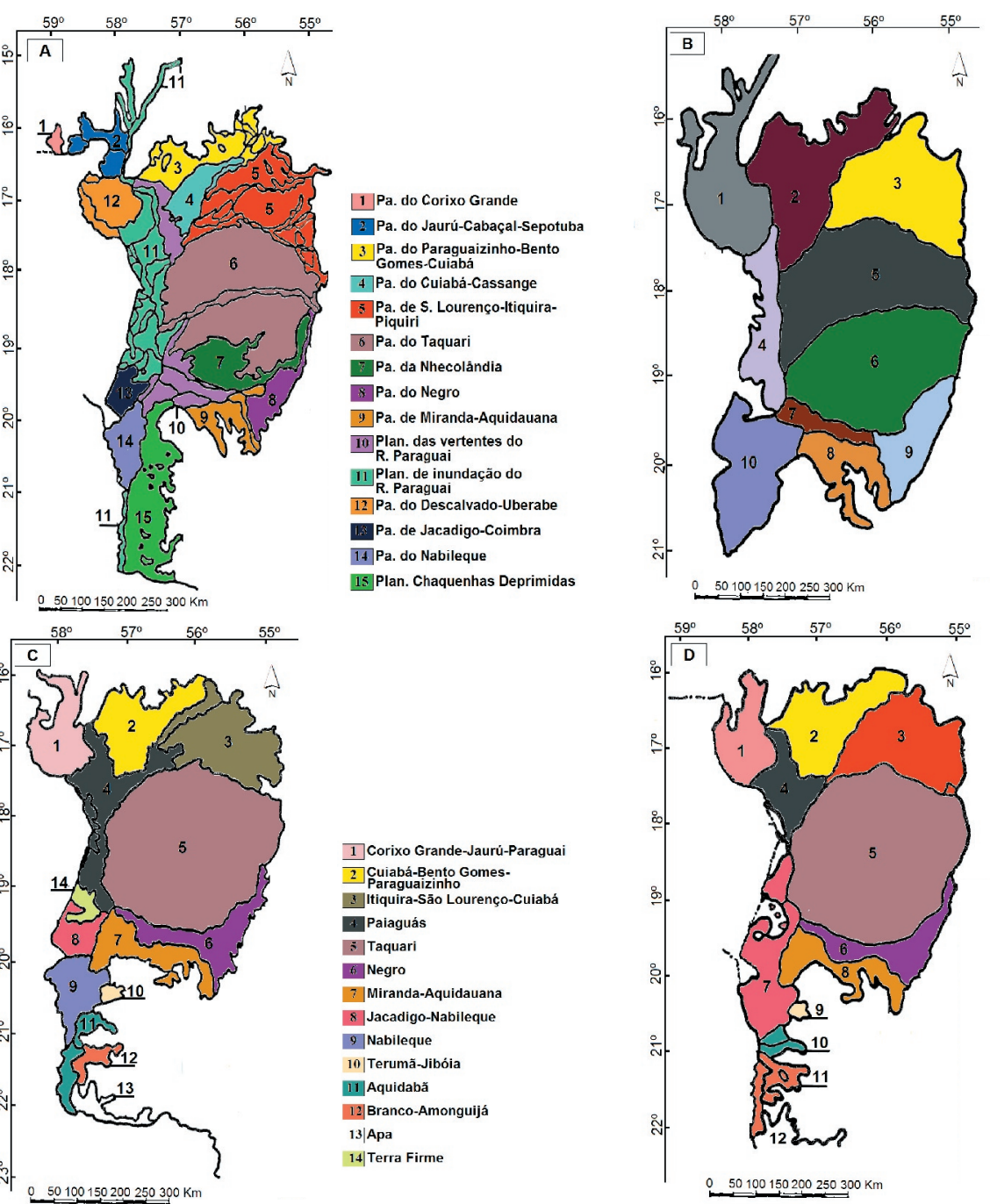

1 Corixo Grande-Jaurú-Paragua 2 Cuiabá-Bento Gomes-

3 |ttiquira-Sāo Lourenço-Guiabả 1. Paiaguás 5|Taquari

(i) Negro

7|Miranda-Aquidauana 8 Jacadigo-Nabileque 9|Nabileque 10|Terumă-Jibóía 11 Aquidabä

12|Branco-Amonguijá

13|Apa

14|Terra Firme
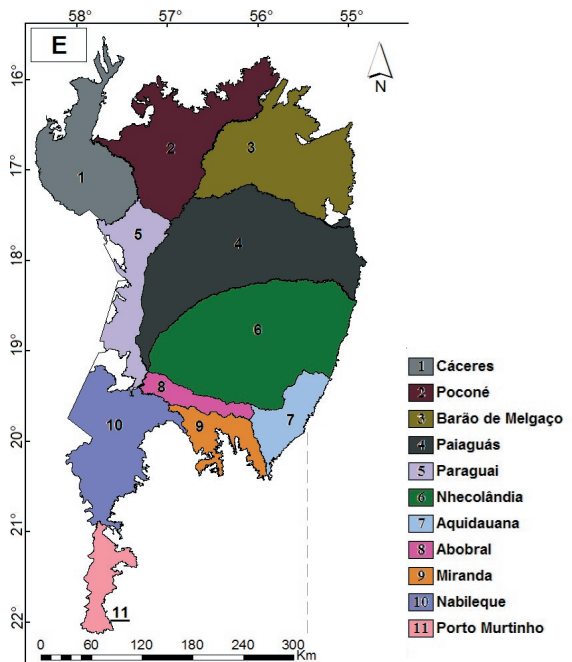

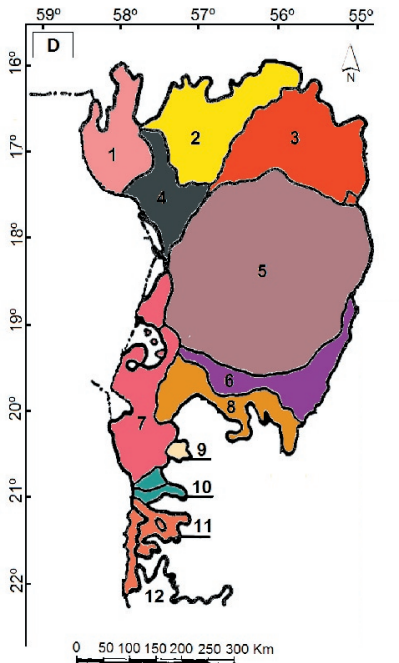

1 Pa. do Corixo Grande-Jaurú2 Pa. do Cuiabá-Bento Gom Paraguaizinho
3 Pa. do Itiquira-S. Lourenço기 Pa. do Paiaguás 5. Pa. do Taquari 6. Pa. do Negro 6. Pa. 7. Pa. do Jacadigo-Nabileque 8 Pa. de Miranda-Aquidauana

9 Pa. do Tarumã Gibóia 10) Pa. do Aquidabã 11 Pa. do Branco Amonguija 12 Pa. do Apa

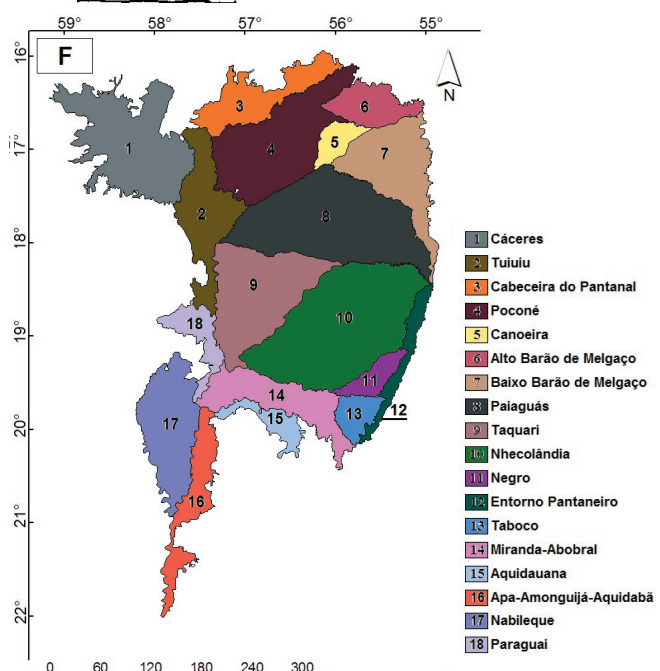

Figura 1. Propostas de subdivisão do Pantanal de acordo com: A) Brasil (1979)* totalizando 15 sub-regiões; B) Adamoli (1982)* com 10 sub-regiões; C) Franco \& Pinheiro (1982)* com 14 sub-regiões; D) Alvarenga et al. (1984)* com 12 subregiões; E) Silva \& Abdon (1998)* com 11 sub-regiões; F) Locks et al. (2011) com 18 sub-regiões. Pantanal (Pa), Planície (Plan.).* Mapas adaptados de Silva (1995).

Figure 1. Subdivisions of the Pantanal according to the sources depicted in legend in Portuguese. 
Pantanal estabelecida e nem quantificação da área. Cabe ressaltar, entretanto, que os mesmos dados deste autor foram utilizados na espacialização de Brasil (1979) (Silva 1995).

Em resumo, as sub-divisões testadas foram: Brasil (1979) com 15 regiões, Adámoli (1982) com 10, Franco \& Pinheiro (1982) com 14, Alvarenga et al. (1984) com 12, Silva \& Abdon (1998) com 11 e Locks et al. (2011) com 18 regiões (Tabela 1).

\section{Dados de distribuição das espécies}

A compilação de dados foi feita, inicialmente, a partir de bases geo-referenciadas de listagens de espécies de mamíferos (Rodrigues et al. 2002) e aves (Tubelis \& Tomas 2001) para o Pantanal. Adicionalmente, foi realizado um levantamento bibliográfico para contemplar a atualização destas listagens. Para tanto foram consultados os bancos de dados: Scielo Brasil, Commonwealth Agricultural Bureau International (CABI), Cambridge, Biological Abstracts, Web of Science, Open J-Gate, The Royal Society Publishing e Armed Forces Pest Management Board (AFPMB) (EUA) Springer, Oxford, BioOne Blackwell Science e Science Direct através do portal Periódicos CAPES (www.periodicos.capes.gov.br). Além das publicações foram consultados os dados de coleções e museus (splink.cria.org.br), bem como de Teses e Dissertações defendidas nas Instituições de Ensino Superior do Brasil (www.dominiopublico.gov.br). As buscas foram realizadas até o período de 30 de abril de 2011. A atualizações dos nomes específicos e redução de sinonímias foram baseadas nas atualizações providas pelo Comitê Brasileiro de Registros Ornitológicos (CRBO 2011) para aves e Reis et al. (2011) para mamíferos. A distribuição destes animais foi obtida de uma base de dados disponível gratuitamente (www.natureserve.org), que possui dados de extensão de ocorrência. Extensão de ocorrência é uma maneira de calcular a área de distribuição geográfica de uma espécie, considerando um polígono delimitado pelos locais com ocorrência dessa espécie (IUCN 2011). Os polígonos foram digitalizados e georreferenciados com base em mapas de espécies oriundo de publicações, corrigindo com informações sobre a extinção das mesmas em certos locais e a origem (se introduzida ou nativa) (Patterson et al. 2007).

Apesar das extensões de ocorrências superestimarem a distribuição das espécies (Araújo 2004), estas extensões são úteis em regiões com alta biodiversidade e baixo esforço de amostragem, como ocorre nas regiões Neotropicais (Bini et al. 2006). Assim, as extensões de ocorrência foram mapeadas em resolução espacial de quadrículas de meio grau (em latitude e longitude), usando como base uma grade de 80 quadrículas que cobrem o Pantanal. Os limite destas quadrículas seguem a articulação internacional ao milionésimo, coincidente com os limites das cartas 1:100000, que é o maior detalhe da cartografia oficial para o Estado do Mato Grosso do Sul.

\section{Análise de dados}

A partir dos dados de extensões de ocorrências foi montada uma matriz de presença e ausência de espécies. Posteriormente, o coeficiente de Jaccard (Koleff et al. 2003) foi empregado para obtenção das matrizes de similaridade entre as quadrículas. As forças de classificações ("classification strength" - CS) das propostas de classificações para o Pantanal foram verificadas pela abordagem de similaridade média (van Sickle \& Hughes 2000). As propostas de classificação do Pantanal foram consideradas nas análises como classificações "a priori". O CS é calculado como a diferença entre a média de todas as similaridades dentro de grupos (W) e a similaridade média entre grupos $(B)(C S=W-B)$. Grandes valores de CS indicam maior força de classificação, ou seja, alta similaridade dentro de sub-regiões e baixa similaridade entre sub-regiões. Posteriormente, as CS foram testadas pelo método de Monte Carlo com 10000 permutações. Foram calculadas as CS para comparar as classificações "a priori" e "a posteriori" (ver abaixo e Padial et al. 2010).

O método de k-means foi empregado para obtenção das classificações a posteriori. O método de agrupamento não hierárquico k-means consiste na partição de um conjunto de objetos em k grupos, no qual os objetos dentro de cada grupo são mais similares entre si que em relação aos objetos dos outros grupos (Legendre \& Legendre 1998). No presente trabalho, o critério de parada para formação dos grupos foi baseado na estatística $R$ (Hartigan 1975):

$$
R=\left(\frac{\operatorname{tr}(W)(k)}{\operatorname{tr}(W)(k+1)}\right)(n-k-1)
$$

onde $\operatorname{tr}(\mathrm{W})$ é o traço de dispersão da matriz dentro dos grupos, n é o número de amostras e $\mathrm{k}$ é o número de grupos. Esta estatística é calculada para diferentes valores de $k$ e os resultados comparados com uma distribuição $F($ g.l. $=P)$. A análise de kmeans foi feita utilizando os escores derivados de uma análise de coordenadas principais (PCoA) aplicados à matriz de similaridade. Para os dados de aves e mamíferos foram retidos 5 e 7 eixos (explicando $\geq 92 \%$ da variação total dos dados), respetivamente, para a posterior aplicação da k-means. O número de grupos k é estabelecido quando o valor da estatística $\mathrm{R}$ passa a ser nãosignificativo. Outra classificação "a posteriori" empregada nas análises foi a utilização do táxon como substituto. Neste caso, os grupos gerados pelo método de k-means para um determinado grupo taxonômico foi utilizado como critério de classificação na análise de CS para o outro táxon.

\section{Resultados}

Os valores das forças de classificações, a despeito de serem significativas, indicaram 
Tabela 2. Forças de classificação para as seis propostas de sub-divisão do Pantanal. B: similaridade média entre grupos, W: similaridade média dentro de cada grupos, CS: forças de classificação, P: níveis de significância baseados em 10000 permutações.

Table 2. Classification strengths (CS) for six proposals of subdivisions of the Pantanal. B: mean similarity between groups, W: mean similarity within groups, $P$ : significance levels based in 10000 permutations.

\begin{tabular}{llccccc}
\hline Classificação & Taxa & Número de classes & B & W & CS & $P$ \\
\hline Adámoli (1982) & Aves & 10 & 0.85 & 0.93 & 0.07 & $<0.001$ \\
& Mamíferos & & 0.90 & 0.96 & 0.06 & $<0.001$ \\
Alvarenga et al. (1984) & Aves & 12 & 0.85 & 0.93 & 0.07 & $<0.001$ \\
& Mamíferos & & 0.89 & 0.95 & 0.06 & $<0.001$ \\
Silva \& Abdon (1998) & Aves & 11 & 0.85 & 0.93 & 0.09 & $<0.001$ \\
& Mamíferos & & 0.89 & 0.96 & 0.07 & $<0.001$ \\
Franco \& Pinheiro (1982) & Aves & 14 & 0.86 & 0.93 & 0.07 & $<0.001$ \\
& Mamíferos & & 0.89 & 0.95 & 0.05 & $<0.001$ \\
Brasil (1979) & Aves & 15 & 0.85 & 0.92 & 0.07 & $<0.001$ \\
& Mamíferos & & 0.89 & 0.95 & 0.05 & $<0.001$ \\
Locks et al. (2011) & Aves & 18 & 0.85 & 0.94 & 0.08 & $<0.001$ \\
\hline
\end{tabular}

que as classificações prévias para o Pantanal apresentaram baixa correspondência com os padrões de diversidade beta de aves e mamíferos (Tabela 2). As propostas de classificação do Pantanal que obtiveram maiores valores de CS foram aquelas propostas por Adámoli (1982), Locks et al. (2011) e Silva \& Abdon (1998), sendo esta última a que apresentou o maior valor de CS, tanto para aves, como para mamíferos.

Altos valores de forças de classificação não foram detectados mesmo quando os agrupamentos foram baseados nos próprios dados de ocorrência das espécies (Tabela 3). As classificações a posteriori, resultantes da k-means, indicaram a formação de 11 grupos para aves e 16 grupos para mamíferos (Figura
2). A utilização dos táxons como grupos substitutos também não resultou em altos valores de forças de classificação, mesmo com valor de significância abaixo de $0.05 \%$. As forças de classificação "a posteriori" também foram maiores para aves em comparação com mamíferos (Tabela 3).

\section{DisCUSSÃO}

Nenhuma das classificações testadas neste estudorepresentouadequadamenteos padrões de distribuição das espécies dos dois grupos de organismos analisados. Assim, somente variáveis fisiográficas não seriam suficientes para delimitar áreas no Pantanal para fins de conservação ou biomonitoramento. Para tanto, seriam necessários inventários faunísticos
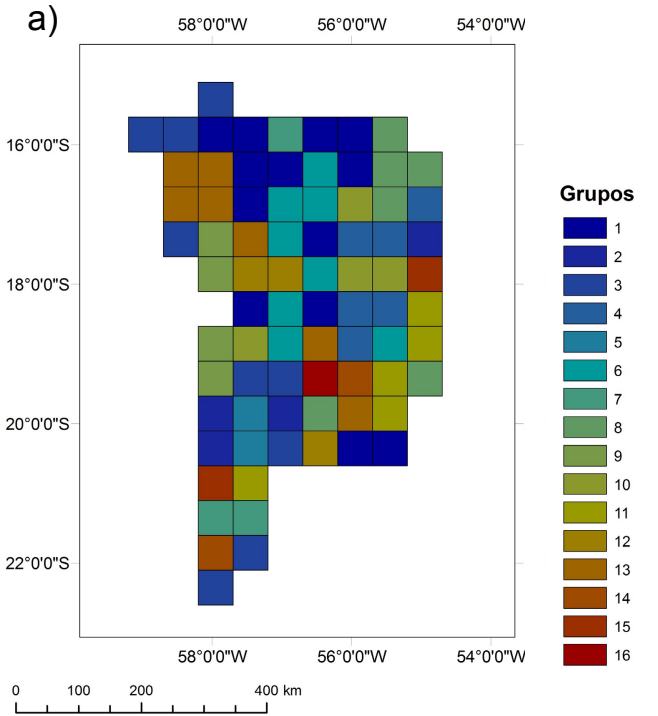

b)

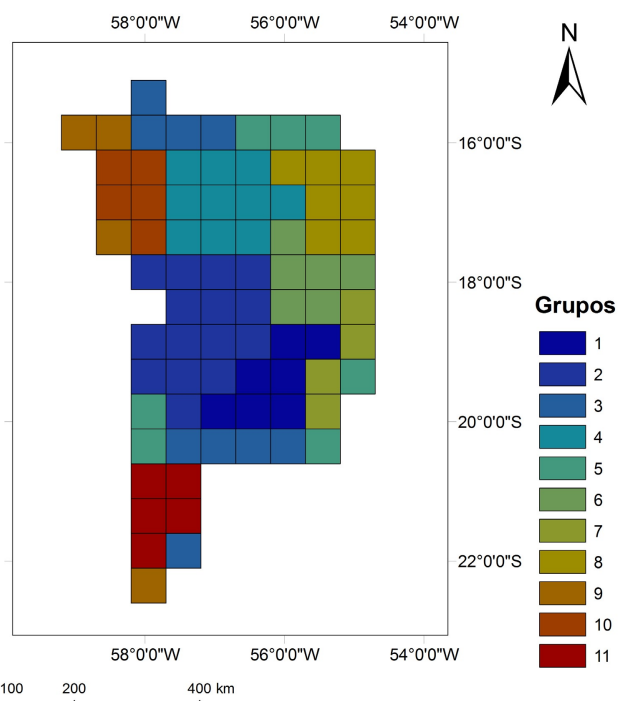

Figura 2. Mapa das classificações "a posteriori" após aplicação de um método de classificação não hierárquico, baseado na distribuição de espécies de mamíferos (a) e aves (b).

Figure 2. The mapping of "a posteriori" Pantanal subdivisions after the application of a non hierarchical classification method, based on data of mammal (a) and bird (b) species distribution. 
Tabela 3. Forças de classificação (CS) para classificações "a posteriori" e utilizando táxon como substituto. B: similaridade média entre grupos, W: similaridade média dentro de cada grupos, $P$ : níveis de significância baseado em 10000 permutações.

Table 3. Classification strengths (CS) for "a posteriori" classifications and using taxa as surrogate. B: mean similarity between groups, $W$ : mean similarity within groups, P: significance levels based in 10000 permutations.

\begin{tabular}{llccccc}
\hline Classificação Taxa & $\begin{array}{c}\text { Número } \\
\text { de } \\
\text { Classes }\end{array}$ & B & W & CS & $P$ \\
\hline a posteriori & Aves & 11 & 0.84 & 0.93 & 0.09 & - \\
& Mamíferos & 16 & 0.89 & 0.94 & 0.05 & - \\
Mamíferos & Aves & 16 & 0.85 & 0.90 & $0.05<0.001$ \\
Aves & Mamíferos & 11 & 0.89 & 0.95 & $0.06<0.001$ \\
\hline
\end{tabular}

detalhados. Como dito anteriormente, as propostas de subdivisão do Pantanal foram baseadas em variáveis fisiográficas obtidas em grandes escalas espaciais e, dados bióticos (com exceção de vegetação) não foram incluídos em nenhuma das propostas existentes. Pelos mesmos motivos, a predição relacionada com as classificações que utilizam como critérios a hidrologia e a vegetação não foi plenamente atendida. As classificações que atendem este critério seriam Adamoli (1982), Alvarenga et al. (1984) e Locks et al. (2011). Como foi observado, destaca-se a performance da classificação de Silva \& Abdon (1998) o menor CS em Locks et. al (2011) para mamíferos. Esse resultado geral é condizente com estudos prévios que mostram que classificações estritamente baseadas em dados fisiográficas nem sempre representam adequadamente os padrões de diversidade beta (Januchowski-Hartley et al. 2011). Uma fraca (e.g., Wright et al. 1998; Heino et al. 2002; Zogaris et al. 2009) bem como uma forte concordância (e.g., Rabeni \& Doisy 2000; Andrew et al. 2011) entre os dados de distribuição de espécies e classificações "a priori" dependem muitas vezes da escala (McDonald et al. 2005; Growns 2009), do táxon analisado (Mora \& Robertson 2005) ou mesmo dos critérios da classificação empregados (Márquez et al. 2001). Resultados semelhantes aos encontrados no presente trabalho foram encontrados para o bioma Cerrado com dados de vertebrados e dois tipos de classificações fisiográficas, no qual o padrão encontrado foi atribuído a respostas a fatores ambientais locais (Padial et al. 2010). Esta explicação para o Pantanal não é totalmente descartada, porém o Pantanal tem uma extensão geográfica muito menor que a do Cerrado. Além disso, a fauna do Pantanal é conhecida por ser generalista e de ampla distribuição geográfica (BrownJunior 1986). Certamente, outros padrões poderiam ser encontrados se dados em escalas mais refinadas estivessem disponíveis. Assim, para testar os efeitos de diferentes escalas nos padrões de correspondência entre esquemas de regionalização e distribuição da fauna, novamente enfatizamos a necessidade de inventários na região do Pantanal.

Mesmo que as diferenças não tenham sido tão grandes entre os CS de aves e mamíferos, foi observado que os resultados de aves foram maioresqueaqueles obtidos para os mamíferos. Para tais diferenças há evidências sob o ponto de vista biológico que podem ser discutidas, principalmente em termos de diferenças nas distribuições das espécies envolvidas. Conforme Brown-Junior (1986), o Pantanal representa barreira para várias espécies de aves como pode servir como corredor a outras. Ou seja, é comum encontrar nas zonas limítrofes do Pantanal, pares e tríades de espécies com fortes relações filogenéticas (com mesmo ancestral comum recente) ao lado de suas respectivas distribuições normais (Amazônia, Cerrado e Chaco), porém ausentes na região central da planície. Em relação a mamíferos, Brown-Junior (1986) ressalta o argumento de Schaller (1983) de que a maioria das espécies é amplamente distribuída nesta parte do continente.

Esquemas de classificação "a posteriori" em comparação com as classificações "a priori" não apresentaram grandes diferenças em relação aos valores das forças de classificação. Este resultado também reforça as conclusões sobre a generalização da distribuição da fauna no Pantanal. Assim, mesmo que as classificações "a posteriori" formem grupos com maiores CS, estes não foram suficientes a ponto de resultar em maior correspondência com os dados faunísticos. Comumente grupos substitutos baseados em dados ambientais (como as classificações fisiográficas do Pantanal) costumam ser menos eficazes quando comparado com grupos substitutos baseados em táxons (ver Rodrigues \& Brooks 2007). Tal comparação entre classificações "a priori" e "a posteriori" é pertinente, pois alguns estudos demonstram que classificações "a posteriori" poderiam ser mais úteis em comparação com as classificações "a priori" em análises de regionalização (Newall \& Wells 2000; Wells et al. 2002). É comum a utilização de um grupo biológico para representar a biodiversidade de outro grupo em planejamento de conservação, e muitas vezes essa abordagem pode implicar em resultados satisfatórios (Caro \& O'Doeherty 1999; Favreau et al. 2006; 
Lovell et al. 2007). O conhecimento sobre as distribuições de aves e mamíferos no Pantanal ainda é insuficiente. No entanto, estes grupos emergem como candidatos às primeiras modelagens de distribuição da fauna para a região. Modelagens baseados em dados biológicos são ferramentas poderosas para tornar mais eficaz o uso de dados esparsos em planejamento regionais de conservação (Ferrier et al. 2002; Pearson et al. 2007). Assim, padrões de distribuição das espécies podem ser inferidos para direcionar onde os esforços de conservação são emergenciais.

Os resultados desse estudo indicam que as classificações isoladamente seriam pouco úteis Devido ao surgimento de novas tecnologias e métodos analíticos é esperado o surgimento de novas propostas de subdivisão fisiográficas para o Pantanal (e.g., Locks et al. 2011), porém ainda persiste a demanda por tratamento diferenciado para atividades produtivas, de manejo, conservação, delimitação e seleção de áreas de pesquisa, bem como para ações normativas ou legislativas (Silva \& Abdon 1998). Ou seja, permaneceria o caso no qual as classificações designariam sub-regiões de uma determinada tipologia, talvez cada vez mais refinadas, porém sem poder preditivo dos padrões de diversidade beta (Brooks et al. 2004). A utilização de características ambientais para mapear as possíveis variações das composições biológicas muitas vezes é uma alternativa quando os recursos financeiros são escassos (Margules \& Pressey 2000; Rodrigues \& Brooks 2007). Contudo, um substituto confiável deve ser capaz de representar a biodiversidade geral (Sarkar \& Margules 2002).

Diversos estudos têm tratado das ameaças sobre o Pantanal (e.g., Willink et al. 2000; Junk \& Cunha 2005; Harris et al. 2005; Harris et al. 2006). Certamente, a ameaça mais preocupante é a perda de habitat decorrente principalmente de desmatamentos, que até o ano de 2004 já acometeu aproximadamente $17 \%$ de sua cobertura vegetal e experimenta taxas anuais de desmatamento que chegam até 2.3\% (Harris et al. 2006). Portanto, é urgente o estabelecimento de planos sistemáticos de conservação com base em dados adequados de distribuição de espécies.

Assim, nenhuma das classificações testadas (a priori) foi eficiente na representação dos padrões de diversidade beta dos dois grupos de organismos analisados. As classificações "a posteriori" geradas pelo método de k-means e a utilização grupo substitutos também foram insuficientes na representação dos padrões. Futuros estudos que melhorem o entendimento da dos fatores que afetam a distribuição das espécies animais no Pantanal são necessários, bem como um esquema de priorização em conservação com dados biológicos mais refinados, visto que o bioma sofre de ameaças graves.

Agradecimentos: R.R.F recebeu bolsa de pós-doutorado do Conselho Nacional de Desenvolvimento Científico e Tecnológico/ CNPq (Processo: 150331/2011-8). F.L.Souza é bolsista do CNPq.

\section{BIBLIOGRAFIA}

ADÁMOLI, J. 1982. O Pantanal e suas relações fitogeográficas com os cerrados: discussão sobre o conceito de complexo do Pantanal. Pp.109-119 em: Anais do 32 ${ }^{\circ}$ Congresso nacional da Sociedade Botânica do Brasil. Teresina-PI, Brasil.

AlHo, CJR. 2008. Biodiversity of the Pantanal: response to seasonal flooding regime and to environmental degradation. Braz. J. Biol., 68(4, Suppl.):957-966.

Alho, CJR. 2011. Biodiversity of the Pantanal: its magnitude, human occupation, environmental threats and challenges for conservation. Braz. J. Biol., 71(1 Suppl.):229-232.

Alvarenga, SM; AE Brasil \& DM Del'Arco. 1982. Geomorfologia. Pp. 125-184 em: BRASIL. Ministério das Minas e Energia. Projeto RADAMBRASIL. Rio de Janeiro-RJ, Brasil.

Alvarenga, SM; AE Brasil; R Pinheiro \& HJH Kux. 1984. Estudo geomorfológico aplicado à Bacia do Alto Paraguai e Pantanais Mato-grossenses. Pp. 89183 em: Projeto RADAMBRASIL. Boletim Técnico, Série Geomorfológica. Salvador-BA, Brasil.

Amaral Filho, ZP. 1986. Solos do Pantanal Matogrossense. Pp. 91-104 em: I Simpósio sobre os recursos naturais e sócio-econômicos do Pantanal. EMBRAPA PANTANAL. Corumbá, MS, Brasil.

ARAújo, MB. 2004. Matching species with reserves - uncertainties from using data at different resolutions. Biol. Conserv., 118:533-538.

Andrew, ME; MA Wulder \& NC Coops. 2011. How do butterflies define ecosystems? A comparison of ecological regionalization schemes. Biol. Conserv., 144(5):1409-1418.

Arponen, A; A Mollanen \& S Ferrier. 2008. A successful community-level strategy for conservation prioritization. J. Appl. Ecol., 45:1436-1445.

Bini, LM; JAF Diniz-Filho; TFLVB Rangel; RP Bastos \& MP PINTo. 2006. Challenging Wallacean and Linnaean shortfalls: knowledge gradients and conservation planning in a biodiversity hotspot. Divers. Distrib., 12:475-482.

BRAsIL. 1979. Estudo de desenvolvimento integrado da bacia do Alto Paraguai: Relatório da $1^{a}$ fase, descrição física e recursos naturais. Ministério do Interior. SUDECO/EDIBAP. Brasília-DF, Brasil. Pp. 235.

BonN, A \& KJ GASTON. 2005. Capturing biodiversity: selecting priority areas for conservation using different criteria. Biodiversity \& Conservation, 14:1083-1100.

Brown-Junior, K. 1986. Zoogeografia da região do Pantanal Mato-grossense. Pp. 137-78 em: I Simpósio sobre os Recursos 
Naturais e Sócio-Econômicos do Pantanal. Corumbá-MS, Brasil.

BRoOKs, T; GAB FonseCA \& ASL Rodrigues. 2004. Species, data, and conservation planning. Conserv. Biol., 18: 1682-1688.

Camargo, G \& EA Fischer. 2005. Primeiro registro do morcego Mimon crenulatum (Phyllostomidae) no Pantanal, sudoeste do Brasil. Biota Neotropica, 5(1):1-4.

Carmignotto, AP. 2004. Pequenos mamíferos terrestres do bioma Cerrado: padrões faunísticos locais e regionais. Tese de Doutorado. Universidade de São Paulo. Brasil.

CARO, TM \& GO DOHERTY. 1999. On the Use of Surrogate Species in Conservation Biology. Conserv. Biol., 13: 805-814.

CAmpos, Z.; M CoutinHo \& W Magnusson. 2003. Terrestrial activity of caiman in the Pantanal, Brazil. Copeia, 3:62834.

CRBO - COMItÊ BRASILEIRO DE Registros ORNITOLÓGICOS. 2011. Listas das aves do Brasil. 10a Edição, 25/1/2011, Disponível em (www.cbro.org.br). Acesso em: 30/04/2011.

CunHA, CN \& WJ Junk. 1999. Composição florística de capões e cordilheiras: localização das espécies lenhosas quanto ao gradiente de inundação no Pantanal de Poconé, MT-Brasil. Pp. 138-143 em: Anais do II Simpósio sobre recursos naturais e sócio-econômicos do Pantanal. Corumbá-MS, Brasil.

Edwards, TC; E Deshler; D Foster \& GG Moisen. 1996. Adequacy of wildlife habitat relation models for estimating spatial distributions of terrestrial vertebrates. Conserv. Biol., 10:263-270.

Favreau, JM; CA Drew; GR Hess; MJ Rubino; FH Koch; ET AL. 2006. Recommendations for assessing the effectiveness of surrogate species approaches. Biodivers. Conserv., 15:3949-3969.

FranCo, MSM \& R PinHeIRO. 1982. Geomorfologia. Pp. 161224 em: BRASIL. Ministério das Minas e Energia. Projeto RADAMBRASIL. Rio de Janeiro-RJ, Brasil.

Ferrier, S; G Watson; J Pearce \& M Drielsma. 2002. Extended statistical approaches to modelling spatial pattern in biodiversity in northeast New South Wales. I. Species-level modelling. Biodivers. Conserv., 11:22752307.

Gonçalves, HC; MA Mercante \& ET Santos. 2011. Hydrological-cycle. Braz. J. Biol., 71(1):233-240.

GROWNS, I. 2009. Differences in bioregional classifications among four aquatic biotic groups: Implications for conservation reserve design and monitoring programs. J. Environ. Manage., 90:2652-2658.

Hartigan, JA. 1975. Clustering Algorithms. New York: Wiley.

Harris, MB; WM Tomas; GM Mourão; CJ Silva; E GUIMARÃES; ET AL. 2005. Desafios para proteger o Pantanal brasileiro: ameaças e iniciativas em conservação. Megadiversidade, 1(1):156-164.

Harris, MC; C Arcângelo; ECT Pinto; G Camargo; MB Ramos-Neto; ET AL. 2006. Estimativa da perda de cobertura vegetal original na Bacia do Alto Paraguai e Pantanal brasileiro: ameaças e perspectivas. Nat. Conservação, 4(2):50-66.

Heino, J; T MuotKa; R PaAvola; H Hämäilä̈Inen \& E KosKENNIEMI. 2002. Correspondence between regional delineations and spatial patterns in macroinvertebrate assemblages of boreal headwater streams. J. North Am. Benthol. Soc., 21(3):397-413.

HeINO, J \& H MYKRÄ. 2006. Assessing physical surrogates for biodiversity: Do tributary and stream type classifications reflect macroinvertebrate assemblage diversity in running waters? Biol. Conserv., 129:418-426.

HuAnG, SL \& JJ Ferng. 1990. Applied land classification for surface water quality management: I. Watershed classification. J. Environ. Manage., 31:107-126.

IUCN. 2011. Guidelines for Using the IUCN Red List Categories and Criteria. Version 9.0. Prepared by the Standards and Petitions Subcommittee. http://goo.gl/Cajfy

Januchowski-Hartley, SR; V Hermoso; RL Pressey; S LINKE; J KOOL; ET AL. 2011. Coarse-filter surrogates do not represent freshwater fish diversity at a regional scale in Queensland, Australia. Biol. Conserv., 144: 2499-2511.

JuNK, WJ \& CJ SILVA. 1999. Conceito do pulso de inundação e suas implicações para o pantanal de Mato Grosso. Pp. 17-28 em: Anais do II Simpósio sobre Recursos Naturais e Sócio-econômicos do Pantanal. Corumbá-MS, Brasil.

Junk, WJ \& CN CunHa. 2005. Pantanal: a large South American wetland at a crossroads. Ecol. Eng., 24:391401.

Junk, WJ; CN Cunha; KM Wantzen; P Petermann; C STRÜMANN; ET AL. 2006. Biodiversity and its conservation in the Pantanal of Mato Grosso, Brazil. Aquat. Sci., 69(3): 278-309.

KolefF, P; JG Gaston \& JJ Lennon. 2003. Measuring beta diversity for presence-absence data. J. Anim. Ecol., 72(3): 367-382.

LeAthwick JR; JMCC Overton \& MMC Leod. 2003. An Environmental Domain Classification of New Zealand and Its Use as a Tool. Conserv. Biol., 17:1612-1623.

Legendre, P \& L Legendre. 1998. Numerical Ecology. 2. ed. Elsevier, Amsterdam. Pp. 853.

Locks, CJ; CL Mioto \& AC Paranhos Filho. 2011. Contribuição do Satélite CBERS-2, Sensor WFI, na delimitação das regiões do Pantanal brasileiro. Anais XV Simpósio Brasileiro de Sensoriamento Remoto. Pp. 3851-3858. .

Longo, JM; EA Fischer; G Camargo \& CF Santos. 2007. Ocorrência de Vampyressa pusilla (Chiroptera, Phyllostomidae) no Pantanal sul. Biota Neotropica, 7:3.

Lourival, R; MB Harris \& JR Montabault. 2000. Introduction to the Pantanal, Mato Grosso do Sul, Brasil. Pp. 28-33 em: Willink, PW; B Chernoff; LE Alonso; JR Montanbault \& R Lourival (eds.). A biological assessment of the aquatic eco-systems of the Pantanal, Mato Grosso do Sul, Brazil, RAP Bul-letin of Biological Assessment 18. Conservation International, Washington, DC.

Lovell, S; M Hamer; R Slotowb \& D Herbert. 2007. Assessment of congruency across invertebrate taxa and taxonomic levels to identify potential surrogates. Biol. Conserv., 139:113-125.

Margules, CR \& RL Pressey. 2000. Systematic conservation planning. Nature, 405:243-253.

Marquéz, AL; R Real \& JM Vargas. 2001. Methods for comparison of biotic regionalizations: the case of pteridophytes in the Iberian Peninsula. Ecography, 24: 659-670.

Mato Grosso doSul. 1989. Macrozoneamento Geoambiental do Estado de Mato Grosso do Sul. Secretaria de Planejamento. Campo Grande: SEPLAN/FIPLAN. Pp. 242.

McDONAld, R; M McKNight; D Weiss; E Selig; M O'CONNOR; ET AL. 2005. Species compositional similarity and ecoregions: Do ecoregion boundaries represent zones of high species turnover? Biol. Conserv., 126:24-40.

Mercante, MA; SC Rodrigues \& JLS Ross. 2011. Geomorphology and habitat diversity in the Pantanal. 
Braz. J. Biol., 71(1):241-253.

Mora, C \& DR Robertson. 2005. Causes of latitudinal gradients in species richness: a test with fishes of the tropical eastern Pacific. Ecology, 86:1771-1782.

MOURÃo, G; ME COUTINHO; RA MAURO;Z CAMPOS; W TOMAS; ET AL. 2000.Aerial surveys of caiman, marsh deer and pampas deer in the Pantanal wetland of Brazil. Biol. Conserv., 92(2):175-183.

Newall, P \& F Wells. 2000. Potential for delineating indicator-defined regions for streams in Victoria, Australia. J. North Am. Benthol. Soc., 19:557-571.

PADIAL, AA; LM BINI;JAF DINIZ-FLLHO; N PEREIRA; RDSOUZA; ET AL. 2010. Predicting Patterns of Beta Diversity in Terrestrial Vertebrates Using Physiographic Classifications in the Brazilian Cerrado. Nat. Conservação, 8:127-132.

Patterson, BD; G Ceballos; W Sechrest; MF Tognelli; T BROOKS; ET AL. 2007. Digital Distribution Maps of the Mammals of the Western Hemisphere. Nature Serve. Virginia, USA. Version 3. www.natureserve.org.

Pearson, RG; RC Jaxworthy; M Nakamura \& AT Peterson. 2007. Predicting species distributions from small numbers of occurrence records: a test case using cryptic geckos in Madagascar. J. Biogeogr., 34:102-117.

Ponce, VM \& CN CunHa. 1993. Vegetated earthmounds in tropical savannas of Central Brazil: a synthesis with special reference to the Pantanal do Mato Grosso. J. Biogeogr., 20:219-225.

RABENI, CF \& KE DoIsy. 2000. Correspondence of stream benthic invertebrate assemblages to regional classification schemes in Missouri. J. North Am. Benthol. Soc., 19(3):419-428.

Reis, NR; AL Peracchi; WA Pedro \& IP Lima (eds.). 2011. Mamíferos do Brasil. Londrina, 2nd ed. Pp. 439.

RODRIGUES, FHG; IM MEdRI; WM TOMAS \& GM MOURão. 2002. Revisão do conhecimento sobre ocorrência e distribuição de Mamíferos do Pantanal. Documento 38. Embrapa Pantanal. Corumbá-MS. Pp. 42.

Rodrigues, ASL \& TM BROOKS. 2007. Shortcuts for biodiversity conservation planning: the effectiveness of surrogates. Annu. Rev. Ecol. Evol. Syst., 38:713-37.

SARKAR, S \& C MARGULES. 2002. Operationalizing biodiversity for conservation planning. J. Biosci., 27(Suppl. 2):299-308.

SANCheZ, RO. 1977. Estudo uviomorfológico del Pantanal; regionalización, sub-regionalización y sectorización geográfico de la depression de la alta cuenca del Rio Paraguai. (S.1.): EDIBAP. Pp. 50.

SANDIN, L \& RK JOHNSON. 2000. Ecoregions and benthic macroinvertebrate assemblages of Swedish streams. J. North Am. Benthol. Soc., 19(3):462-474.

SCHALLER, GB. 1983. Mammals and their biomass on a Brazilian ranch. Arq. Zool., 31:1-36.

VAN SiCKLE, J \& RM Hughes. 2000. Classification strengths of ecoregions, catchments and geographic clusters for aquatic vertebrates in Oregon. J. North Am. Benthol. Soc., 19(3):370-384.

SILVA, JSV. 1995. Elementos fisiográficos para delimitação do Ecossistema Pantanal: Discussão e proposta. Oecologia Brasiliensis, 1:439-458.

SILVA, JSV \& MM Abdon. 1998. Delimitação do Pantanal Brasileiro e suas sub-regiões. Pesqui.Agropecu. Bras., 33: 1703-1711.

Silva, MP; RA Mauro; GM Mourão \& M CoutinHo. 2000. Distribuição e quantificação de classes de vegetação do Pantanal através de levantamento aéreo. Rev. Bras. Bot. 23(2):143-152.

Tubelis, DP \& WM Tomas. 2003. Birds species of the Pantanal wetland, Brazil. Ararajuba, 11:5-37.

Willink, PW; B Chernoff; LE Alonso; JR Montabault \& R Lourival. 2000. A biological assessment of the aquatic eco-systems of the Pantanal, Mato Grosso do Sul, Brazil. RAP Bulletin of Biological Assessment 18. Conservation International, Washington, DC. Pp. 305.

Wells, F; L Metzeling \& P Newall. 2002. Macroinvertebrate regionalization for use in the management of aquatic ecosystems in Victoria, Australia. Environ. Monit.Assess., 74:271-294.

Wright, RG; MP MurRAy \& T MERRILl. 1998. Ecoregions as a level of ecological analysis. Biol. Conserv., 86:207-213.

Zogaris, S; NA Economou \& P Dimopoulos. 2009. Ecoregions in the Southern Balkans: Should Their Boundaries Be Revised? Environ. Manage., 43:682-697. 